\title{
MATHEMATICAL MODELING OF NONSTATIONARY SEPARATION PROCESSES IN GAS CENTRIFUGE CASCADE FOR SEPARATION OF MULTICOMPONENT ISOTOPE MIXTURES
}

\author{
Alexey Orlov ${ }^{1}$, Anton Ushakov ${ }^{2, *}$, and Victor Sovach ${ }^{2}$ \\ ${ }^{1}$ National Research Tomsk Polytechnic University, 634050 Tomsk, Russia \\ ${ }^{2}$ SC "PA Electrochemical Plant”, 663690 Zelenogorsk, Russia
}

\begin{abstract}
This article presents results of development of the mathematical model of nonstationary separation processes occurring in gas centrifuge cascades for separation of multicomponent isotope mixtures. This model was used for the calculation parameters of gas centrifuge cascade for separation of germanium isotopes. Comparison of obtained values with results of other authors revealed that developed mathematical model is adequate to describe nonstationary separation processes in gas centrifuge cascades for separation of multicomponent isotope mixtures.
\end{abstract}

\section{Introduction}

Nonstationary processes can appear in the operation of gas centrifuge (GC) cascade for separation of multicomponent isotope mixtures (MCIM). During these processes isotope composition changes in cascade and it is necessary to minimize losses of cascade productivity. At present, there are mathematical models of nonstationary separation processes $[1,2]$, which have such disadvantage as a limited field of application: simulation is only possible in the case of stationary hydraulic parameters of GC cascade. Using of these models is impossible for the joint calculation of nonstationary hydraulic and separation processes. For elimination of this disadvantage we firstly developed the mathematical model of nonstationary hydraulic processes in GS cascade for MCIM separation [3]. This model was tested for separation $\mathrm{Si}, \mathrm{Xe}, \mathrm{W}, \mathrm{Ni}$ isotopes. Further studies were aimed at creating the mathematical model of nonstationary separation processes in GC cascade for the MCIM separation. This article is devoted to its description.

* Corresponding author: ushakovaa2015@,sibmail.com 


\section{The description of the mathematical model}

MCIM separation takes place into a cascade (fig. 1). MCIM is composed of $n$ components identified by $j$ index $(j=\overline{1, N})$. A cascade consists of $S$ separation stages numbered by $i$ index $(i=\overline{1, S})$ and connected by counter-current type [4]. The cascade has three flows: input feed flow $F$, output light fraction flow $P$ and heavy fraction flow $W$.

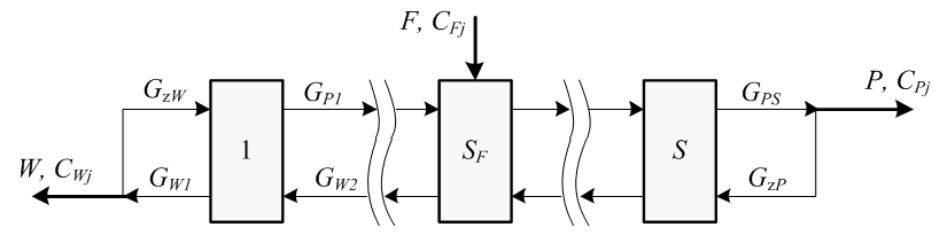

Fig. 1. Separation cascade.

During stationary hydraulic process the flows and concentrations of components in stage are interrelated by material balance equations and balance equations of each component:

$$
\begin{gathered}
F=P+W \\
F C_{F j}=P C_{P j}+W C_{W j}
\end{gathered}
$$

The stage has three flows: incoming feed flow $G_{F i}$, output light fraction flow $G_{P i}$ and heavy fraction flow $G_{W i}$ :

$$
\begin{gathered}
G_{F i}=G_{P i}+G_{W i} \\
G_{F i} C_{F i j}=G_{P i} C_{P i j}+G_{W i} C_{W i j}
\end{gathered}
$$

The separation of components in the $i$ th stage is given by:

$$
\chi_{i j l}=\frac{C_{P i j} C_{W i l}}{C_{P i l} C_{W i j}}
$$

where $\chi_{i j l}$ is a separation factor of the $j$ th and $l$ th components in the $i$ th stage.

For square cascade there are dimensionless parameters [1, 4]:

$$
\begin{gathered}
y_{P}=S \ln \chi_{0} \\
\tau=\frac{G_{F} t \ln ^{2} \chi_{0}}{H^{\prime}}
\end{gathered}
$$

where $y_{P}$ is cascade length; $\chi_{0 i}$ is separation factor per unit difference between the mass numbers; $\tau$ is time; $H^{\prime}$ is cascade holdup, $\mathrm{kg}$; $t$ is time, $\mathrm{s}$.

The method of calculation of the nonstationary separation processes is based on the following points:

- Each stage is composed of finite number of capacities. MCIM is distributed in these capacities.

- At any time the value of process gas temperature, pressure and concentrations of each component are the same at any points of capacity. 
- Basic equations of the mathematical model are the balance equations of process gas and components in each capacity and the equation of MCIM separation at the GC.

In the regards, the holdup of cascade is distributed in $4 S$ capacities (fig. 2).

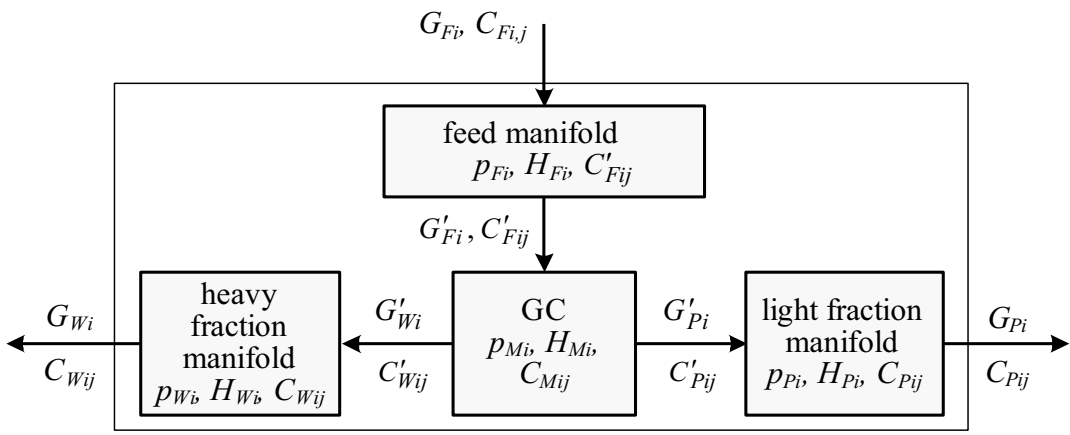

Fig. 2. Capacities of $i$ th stage.

Differential equation describing nonstationary separation processes in each capacity is written in the general form as:

$$
\frac{d\left(H C_{j}\right)}{d t}=G_{I N} C_{I N j}-G_{O U T} C_{j}
$$

Replacing derivatives to difference equations by backward Euler method [5], we have received:

$$
\begin{gathered}
\frac{H^{(k)} C_{j}^{(k)}-H^{(k-1)} C_{j}^{(k-1)}}{\Delta t}=G_{I N}^{(k)} C_{I N j}^{(k)}-G_{O U T}^{(k)} C_{j}^{(k)} \\
\Delta t=t^{(k)}-t^{(k-1)}
\end{gathered}
$$

where $\Delta t$ is time step; $k$ is the index of time layer.

The system of linear algebraic equations received from equations (10), is solved by the Gaussian elimination [5]. The result of calculation is values of component concentrations in each capacities and flows at every time layers. We have completed the algorithm described herein as a software product, developed on Delphi programming language using Embarcadero Delphi XE2.

\section{The results of calculation cascade for germanium isotope separation}

We have calculated parameters of nonstationary separation process in model GC cascade for germanium isotope separation for the purpose of verification of developed mathematical model and created software product. The two initial conditions are:

1. Hydraulic parameters of cascade correspond to the stationary values, isotope concentrations in all stage are equal to their concentrations in feed flow [1,2] (initial conditions 1).

2. Hydraulic parameters of cascade and isotope concentrations in all stage are the result of calculation of the nonstationary process of a filling cascade with process gas (initial conditions 2) (fig. 3). 
The calculation results of nonstationary separation process in the cascade for germanium isotope separation are given on fig. 4 .

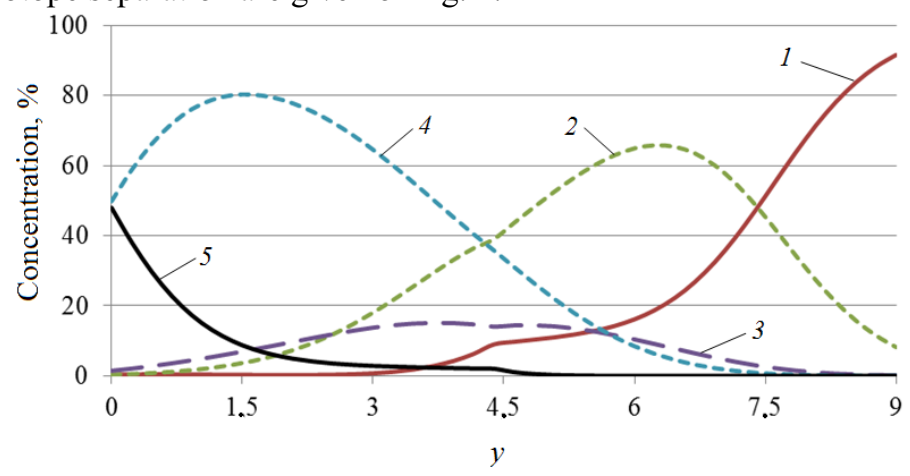

Fig. 3. Isotopes distribution along length of cascade after filling cascade by process gas: $1-{ }^{70} \mathrm{Ge}, 2-$ ${ }^{72} \mathrm{Ge}, 3-{ }^{73} \mathrm{Ge}, 4-{ }^{74} \mathrm{Ge}, 5-{ }^{76} \mathrm{Ge}$.

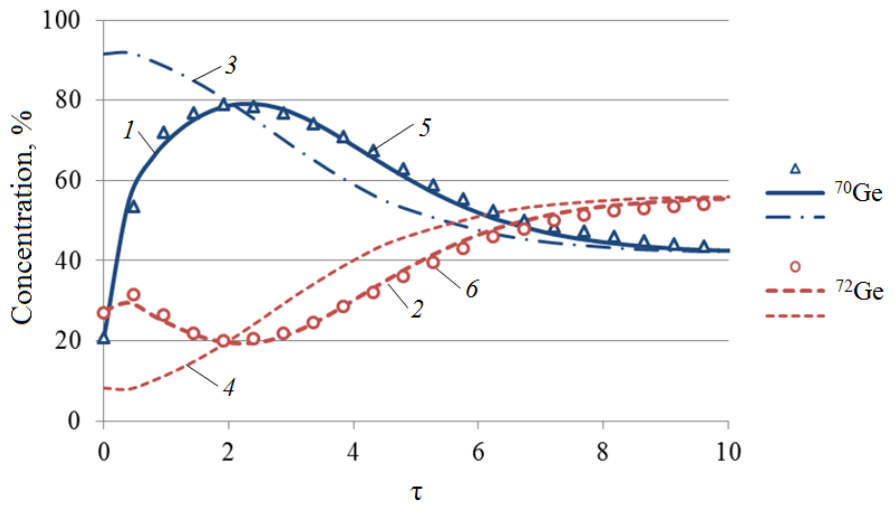

Fig. 4. Time variation of ${ }^{70} \mathrm{Ge}$ and ${ }^{72} \mathrm{Ge}$ concentrations in light fraction flow of cascade: $1,2-$ developed model (initial conditions 1); 3, 4-developed model (initial conditions 2); 5, 6-models of other researchers $[1,2]$.

As it's seen on fig. 4, the calculation results obtained by developed model for initial conditions 1 (the curves 1 and 2) are well correlated with the results of other researchers [6, 7], which demonstrates the adequacy of developed mathematical model.

\section{Conclusion}

1. The result of research is creation of mathematical model of nonstationary separation processes in gas centrifuge cascade for separation of multicomponent isotope mixtures, representing the differential equation system of first order with given initial conditions.

2. The solution algorithm of equation system describing nonstationary separation processes is developed and realized as a software product.

3. We have made the calculation parameters of nonstationary separation process in model GC cascade for germanium isotope separation for the purpose of verification of developed mathematical model and created software product. Comparison of obtained values with results of other authors revealed that developed mathematical model is adequate to describe nonstationary separation processes in gas centrifuge cascades for separation of multicomponent isotope mixtures. 
4. This mathematical model together with model of nonstationary hydraulic processes [9] makes it possible to perform calculations of isotope concentrations in cascade stages during its filling by process gas.

\section{References}

1. N.I. Laguntcov, E.V. Levin, B.I. Nikolaev, G.A. Sulaberidze, At. Energ. 6, 62 (1987)

2. G.A. Sulaberidze, V.A. Palkin, V.D. Boricevich, V.D. Borman, A.V. Tichomirov Theory of cascades for separation of binary and multicomponent isotope mixtures (MEPhI, Moscow, 2011)

3. A.A. Orlov, V.P. Sovach, A.A. Ushakov Altern. Energ. Ekol. 23, 187 (2015)

4. K. Cohen The theory of isotope separation as applied to the large scale production of U-235 (McGraw Hill, New York, 1951)

5. U.G. Pirumov Numerical methods (Drofa, Moscow, 2003) 